\title{
A Learning Approach For Prioritized Handoff Channel Allocation In Mobile Multimedia Networks
}

El-Alfy, E.-S. Yu-Dong Yao Heffes, H.;Dept. of Comput. Sci., King Fahd Univ. of Pet. \& Miner., Dhahran;

Wireless Communications, IEEE Transactions on;Publication Date: July2006;Vol:

5,Issue: 7

King Fahd University of Petroleum \& Minerals

http://www.kfupm.edu.sa

Summary

An efficient channel allocation policy that prioritizes handoffs is an indispensable ingredient in future cellular networks in order to support multimedia traffic while ensuring quality of service requirements (QoS). In this paper we study the application of a reinforcement-learning algorithm to develop an alternative channel allocation scheme in mobile cellular networks that supports multiple heterogeneous traffic classes. The proposed scheme prioritizes handoff call requests over new calls and provides differentiated services for different traffic classes with diverse characteristics and quality of service requirements. Furthermore, it is asymptotically optimal, computationally inexpensive, model-free, and can adapt to changing traffic conditions. Simulations are provided to compare the effectiveness of the proposed algorithm with other known resource-sharing policies such as complete sharing and reservation policies

For pre-prints please write to:abstracts@kfupm.edu.sa 